\title{
Revisiting tuberculous pleurisy: pleural fluid characteristics and diagnostic yield of mycobacterial culture in an endemic area
}

\author{
Sheng-Yuan Ruan, ${ }^{1}$ Yu-Chung Chuang, ${ }^{1}$ Jann-Yuan Wang, ${ }^{1}$ Jou-Wei Lin, ${ }^{2}$ \\ Jung-Yien Chien, ${ }^{3}$ Chun-Ta Huang, ${ }^{1}$ Yao-Wen Kuo, ${ }^{2}$ Li-Na Lee, ${ }^{1}$ Chong-Jen $\mathrm{J} \mathrm{Yu}$
}

- Additional materials are published online only. To view these files please visit the journal online (http://dx.doi.org/ 10.1136/thoraxjnl-2011201363)

${ }^{1}$ Department of Interna Medicine, National Taiwan University Hospital and College of Medicine, Taipei, Taiwan ${ }^{2}$ Department of Internal Medicine, National Taiwan University Hospital Yun-Lin Branch, Yun-Lin, Taiwan ${ }^{3}$ Department of Health, Chest Hospital, Tainan, Taiwan

\section{Correspondence to} Dr Jann-Yuan Wang, Department of Internal Medicine, National Taiwan University Hospital, No. 7 , Chung-Shan South Rd, Taipei 10002, Taiwan; jywang@ntu.edu.tw

Received 12 November 2011 Accepted 3 March 2012

Published Online First

21 March 2012

\section{UN OCKT:}

This paper is freely available online under the BMJ Journals unlocked scheme, see http:// thorax.bmi.com/site/about/ unlocked.xhtml

\begin{abstract}
Background Tuberculous pleurisy is traditionally indicated by extreme lymphocytosis in pleural fluid and low yield of effusion culture. However, there is considerable inconsistency among previous study results. In addition, these data should be updated due to early effusion studies and advances in culture methods. Methods From January 2004 to June 2009, patients with tuberculous pleurisy were retrospectively identified from the mycobacteriology laboratories and the pathology and tuberculosis registration databases of two hospitals in Taiwan where tuberculosis is endemic. Pleural fluid characteristics and yields of mycobacterial cultures using liquid media were evaluated.
\end{abstract}

Results A total of 382 patients with tuberculous pleurisy were identified. The median lymphocyte percentage of total cells in pleural fluids was $84 \%$ (IQR $64-95 \%$ ) and $17 \%$ of cases had a lymphocyte percentage of $<50 \%$. The lymphocyte percentage was negatively associated with the probability of a positive effusion culture (OR $0.97 ; 95 \% \mathrm{Cl} 0.96$ to 0.99 ). The diagnostic yields were $63 \%$ for effusion culture, $48 \%$ for sputum culture, $79 \%$ for the combination of effusion and sputum cultures, and $74 \%$ for histological examination of pleural biopsy specimens.

Conclusion The degree of lymphocyte predominance in tuberculous pleurisy was lower than was previously thought. The lymphocyte percentage in pleural fluid was negatively associated with the probability of a positive effusion culture. With the implementation of a liquid culture method, the sensitivity of effusion culture was much higher than has been previously reported, and the combination of effusion and sputum cultures provided a good diagnostic yield.

\section{INTRODUCTION}

Tuberculous pleurisy is the second most common form of extrapulmonary tuberculosis $(\mathrm{TB})^{12}$ and a common cause of pleural effusion in endemic TB areas. ${ }^{34}$ A definitive diagnosis of tuberculous pleurisy usually requires mycobacterial culture of pleural fluid, pleural biopsy or other diagnostic tests. ${ }^{5}{ }^{6} \mathrm{~A}$ high index of suspicion from a pleural fluid analysis is essential for diagnosis. However, the traditional concepts about the pleural fluid findings in tuberculous pleurisy mainly come from studies conducted decades ago that have not been validated in current practice. ${ }^{7-10}$ Pleural fluid has been frequently studied owing to the popular use of

\section{Key messages}

What is the key question?

- Tuberculous pleurisy is traditionally indicated by extreme lymphocytosis in pleural fluid and low yield of effusion culture. However, the results of previous studies in this field vary widely and their applicability to present practice has not been evaluated.

What is the bottom line?

- The degree of lymphocyte predominance in tuberculous pleurisy is lower than was previously thought. With the implementation of a liquid culture method, the yield of effusion mycobacterial culture is much higher than previously reported. High lymphocyte percentage in pleural fluid is negatively associated with the probability of a positive effusion culture.

\section{Why read on?}

- This study provides updated data on the pleural fluid characteristics and yield of effusion culture in the diagnosis of tuberculous pleurisy. The traditional concept about tuberculous pleurisy cannot be totally applied to current practice.

ultrasound, which may modify the pleural fluid findings at disease presentation. ${ }^{011}$ In addition, the data from previous studies on the diagnostic yields for tuberculous pleurisy have varied widely and are not consistent. These studies have suggested the following diagnostic yields: $12-70 \%$ for effusion culture, ${ }^{9} 120-52 \%$ for sputum culture ${ }^{12} 13$ and $50-90 \%$ for histological examination of pleural biopsy specimens. ${ }^{14-17}$ We consider that variations in the study results are related to the patient selection criteria, the small number of cases in most of the studies and variable TB prevalence. Furthermore, advances in culture techniques have improved the yield of mycobacterial culture in this disease. Liquid culture media have been shown to have higher yields and faster results than solid media. ${ }^{18} 19$

The objective of the present study was to provide updated information about the pleural fluid characteristics and diagnostic yields of mycobacterial culture and pleural biopsy. Differences between the cases with positive and negative effusion 
mycobacterial cultures were compared to determine the predictors of a positive effusion culture.

\section{METHODS \\ Study setting}

This was a retrospective cohort study conducted at the National Taiwan University Hospital and the National Taiwan University Hospital Yun-Lin Branch, two university-affiliated teaching hospitals in Taiwan. Taiwan is an endemic area of TB, with an incidence of 62-74 per 100000 population from 2004 to $2008 .^{20}$ The National Tuberculosis Program of Taiwan requires that all cases of culture-confirmed TB or clinically suspicious TB should be reported to the Taiwan Centers for Disease Control. Such cases are also recorded in the $\mathrm{TB}$ registration databases of the two study hospitals. Because of the high incidence of TB, effusion mycobacterial culture is a routine examination for all undiagnosed pleural effusions regardless of the clinical presentation and pleural fluid findings in both study hospitals. This study was approved by the Research Ethics Committee of the National Taiwan University Hospital.

\section{Diagnosis of tuberculous pleurisy}

Pleural effusion was diagnosed as tuberculous according to the following criteria: positive mycobacterial culture in pleural fluid or pleural biopsy tissue samples; granulomatous inflammation in pleural biopsy tissue samples; positive mycobacterial culture of sputum samples and pleural effusion that were resolved with anti-TB treatment; or clinically suspected tuberculous pleurisy and pleural effusion that were resolved with anti-TB treatment. In the fourth criterion, 'clinical suspicion' means that these patients had no systemic diseases, and were excluded from malignancy and other pleural diseases. Moreover, there were suggestive clues of TB from contact history, radiographical findings or clinical presentation.

We obtained and merged the lists of patients with tuberculous pleurisy or other tuberculosis diseases with concurrent pleural effusion from the following three sources: our TB registration database; our mycobacteriology laboratory database to identify cases with a positive TB culture in any specimen; and our pathology database to identify cases with granulomatous inflammation in pleural tissue specimens. The study period was from January 2004 to June 2009. The identified cases were further evaluated if they satisfied one of the four criteria. All cases that failed to satisfy one of these criteria were excluded from the study. Patients with TB empyema, defined as pus-like appearance of pleural fluid or positive acid-fast bacilli (AFB) smear on pleural fluid, were also excluded.

\section{Data extraction}

Medical records were reviewed to obtain data, including age, gender, comorbidities, date of the first visit for pleural effusion, date of initiation of anti-TB treatment and date of the last visit. The laboratory data included initial blood cell counts, renal function tests, liver function tests, protein levels, lactate dehydrogenase (LDH) levels, glucose levels in serum and pleural fluid, and white blood cell and differential cell counts of pleural fluid. Chest radiographs were reviewed by a pulmonologist and a radiologist to assess the initial radiographical presentation and response to treatment. The results of the AFB smears and the mycobacterial cultures of pleural fluid and sputum were obtained.

\section{Bacteriological procedures}

The AFB smears were performed with auramine-rhodamine fluorochrome and examined using standard procedures. ${ }^{21}$ The specimens for the mycobacterial cultures were processed using the standard $\mathrm{N}$-acetyl-L-cysteine and sodium hydroxide method. The mycobacterial cultures were performed by inoculating $0.5 \mathrm{ml}$ of the processed specimens into MGIT 960 culture tubes according to the manufacturer's instructions (BACTEC Mycobacteria Growth Indicator Tube 960 System, BD Diagnostic Instrument Systems, Sparks, Maryland, USA). The fluorometric BACTEC technique was used to detect positive tubes for a maximum of 6 weeks. For the positive tubes, a subculture was made on Middlebrook 7H11 selective agar (Remel Inc, Lexena, Kansas, USA).

\section{Statistical analysis}

The categorical variables were compared using the $\chi^{2}$ test or Fisher's exact test, as appropriate, while the continuous variables were compared using t tests or the Mann-Whitney $U$ test. A multivariate logistic regression analysis was conducted to identify the predictors of a positive effusion mycobacterial culture. The analysed variables included age, gender, underlying diseases, effusion levels of protein, LDH levels, glucose levels, white blood cell counts and the lymphocyte percentages of the effusions. Variables with a $p$ value of $<0.2$ in the univariate regression were added in a stepwise manner and selected to determine the final model. A generalised additive model was used to assess the linearity of the relationship between effusion culture and parameters of interest. ${ }^{22}$ The time-to-event curves for each variable were generated using the Kaplan-Meier method and compared by the log-rank test. The data were analysed using Stata software, V.10 (StataCorp).

\section{RESULTS}

\section{Cases of tuberculous pleurisy}

There were 3263 patients with definite or suspicious TB in the study period. Of these patients, 792 patients had tuberculous pleurisy or another form of TB disease with concurrent pleural effusion. Three patients with positive AFB smears on their pleural fluids were considered to have tuberculous empyema and excluded for analysis. Among the 789 patients, 382 patients were diagnosed as having tuberculous pleurisy, including 241 with effusion cultures positive for Mycobacterium tuberculosis and 141 with negative effusion cultures. In the 141 patients with negative effusion cultures, 48 received a diagnosis by pleural biopsy, 45 had positive sputum cultures and had pleural effusion that resolved with anti-TB treatment, and 48 were clinical suspicions and had pleural effusion that resolved with anti-TB treatment. Table 1 summarises the characteristics of the 382 patients.

\section{Pleural fluid findings}

Table 2 shows the laboratory data for these patients. In general, the patients had no leukocytosis but had an elevated serum level of $\mathrm{LDH}$. Protein-rich exudate was a common feature of the pleural fluid analysis. Figure 1A shows the distribution of the effusion lymphocyte percentages. A lymphocyte percentage of $<50 \%$ was observed in $17 \%$ of the patients. The effusion cell differential of the three excluded patients with TB empyema showed $93 \%, 92 \%$ and $6 \%$ neutrophils. The case with $6 \%$ neutrophils had been treated with levofloxacin before thoracentesis.

Compared with the culture-negative effusions, the culturepositive effusions had a lower lymphocyte percentage (median $92 \%$ vs $78 \%, p<0.001)$. The probability of a positive effusion culture was negatively associated with the effusion lymphocyte 
Table 1 Characteristics of 382 patients with tuberculous pleurisy

\begin{tabular}{|c|c|c|c|c|}
\hline \multirow[b]{2}{*}{ Characteristics } & \multirow[b]{2}{*}{ n (\%) } & \multicolumn{2}{|c|}{ Pleural effusion culture, $\mathbf{n}(\%)$} & \multirow[b]{2}{*}{ p Value } \\
\hline & & Positive, $n=241$ & Negative, $n=141$ & \\
\hline Age (mean $\pm \mathrm{SD}$ ) & $62 \pm 20$ & $63 \pm 21$ & $60 \pm 19$ & $0.20^{*}$ \\
\hline Gender (men/women) & $270 / 112$ & $173 / 68$ & $97 / 44$ & 0.54 \\
\hline Visit to diagnosis, median (IOR) & $15(7-29)$ & $17(8-30)$ & $14(6-27)$ & $0.08 \dagger$ \\
\hline \multicolumn{5}{|l|}{ Underlying diseases } \\
\hline COPD or current smoker & $92(24)$ & $54(22)$ & $38(27)$ & 0.32 \\
\hline Diabetes mellitus & 73 (19) & $52(22)$ & $21(15)$ & 0.11 \\
\hline Cancer & $54(14)$ & $45(19)$ & $9(6)$ & $<0.001$ \\
\hline Renal replacement therapy & $33(9)$ & $28(12)$ & $5(4)$ & 0.007 \\
\hline Heart failure & $23(6)$ & $19(8)$ & $4(3)$ & 0.05 \\
\hline Past history of tuberculosis & $17(4)$ & $8(3)$ & $9(6)$ & 0.16 \\
\hline Liver cirrhosis & $16(4)$ & $14(6)$ & $2(1)$ & 0.04 \\
\hline Autoimmune diseases & $10(3)$ & $8(3)$ & $2(1)$ & 0.34 \\
\hline HIV seropositive & $8(2)$ & $6(2)$ & $2(1)$ & 0.72 \\
\hline Post transplantation & $4(1)$ & $3(1)$ & $1(1)$ & 1.00 \\
\hline \multicolumn{5}{|l|}{ Effusion site $\ddagger$} \\
\hline Right & $237(62)$ & 147 & 90 & 0.46 \\
\hline Left & 149 (39) & 98 & 51 & \\
\hline
\end{tabular}

percentage (figure $1 \mathrm{~B}$ ). The multivariate logistic regression model identified four independent predictors of the effusion culture results, including cancer as an underlying disease (OR 2.66; $95 \%$ CI 1.14 to 6.19), effusion lymphocyte percentage (OR $0.97 ; 95 \%$ CI 0.96 to 0.99 ), effusion protein level (g/dl) (OR 0.6; $95 \%$ CI 0.45 to 0.79 ) and effusion LDH level (U/litre) (OR 1.001; $95 \%$ CI 1.00 to 1.002$)$.

\section{Diagnostic yields}

The yields of effusion culture, sputum culture and pleural biopsy in the diagnosis of tuberculous pleurisy are summarised in table 3. The diagnostic yields were $63 \%$ and $48 \%$ for the effusion and sputum cultures, respectively. A combination of the effusion and sputum cultures achieved a diagnostic sensitivity of $79 \%$. In contrast, the diagnostic yield was 74\% for histological examination of pleural biopsies.

\section{Outcome}

Figure 2 shows the 180-day survival curve of the patients with positive and negative effusion cultures. The probability of survival significantly differed according to the effusion culture results (log-rank test, $\mathrm{p}<0.001)$. In the patients with positive effusion cultures, there were 21 (9\%) deaths before the time of diagnosis.

\section{DISCUSSION}

This study reveals several important findings regarding the diagnosis of tuberculous pleurisy. The diagnostic yield of

Table 2 Biochemical characteristics and cells differential of 382 tuberculous pleurisy cases

\begin{tabular}{|c|c|c|c|c|c|c|}
\hline \multirow[b]{2}{*}{ Characteristic } & \multirow[b]{2}{*}{$\mathbf{n}$} & \multirow[b]{2}{*}{ Mean \pm SD } & \multirow[b]{2}{*}{ Median (IOR) } & \multicolumn{2}{|l|}{ Effusion culture } & \multirow[b]{2}{*}{ p Value } \\
\hline & & & & $\begin{array}{l}\text { Positive, mean } \pm \text { SD } \\
\text { or median (IOR) }\end{array}$ & $\begin{array}{l}\text { Negative, mean } \pm S D \\
\text { or median (IOR) }\end{array}$ & \\
\hline \multicolumn{7}{|l|}{ Blood test } \\
\hline Platelet $(K / \mu \mathrm{l})$ & 345 & $282 \pm 114$ & $272(208-347)$ & $262 \pm 115$ & $315 \pm 106$ & $<0.001$ \\
\hline Protein (g/dl) & 171 & $6.8 \pm 1$ & $6.8(6.1-7.5)$ & $6.6 \pm 1$ & $7.1 \pm 1$ & 0.003 \\
\hline LDH (U/litre) & 149 & $565 \pm 271$ & $489(413-644)$ & $509(416-660)$ & $453(379-578)$ & $0.03^{*}$ \\
\hline Lymphocyte \% & 367 & $76 \pm 25$ & $84(64-95)$ & $78(55-91)$ & $92(82-97)$ & $<0.001^{*}$ \\
\hline Neutrophil \% & 367 & $15 \pm 21$ & $5(1-19)$ & $9(2-28)$ & $2(1-8)$ & $<0.001^{*}$ \\
\hline Protein $(\mathrm{g} / \mathrm{d} \mathrm{l})$ & 362 & $4.8 \pm 1$ & $5(4.2-5.4)$ & $4.6 \pm 1.1$ & $5 \pm 0.8$ & $<0.001$ \\
\hline LDH (U/litre) & 362 & $988 \pm 1259$ & $680(455-1055)$ & $849(500-1313)$ & $550(389-763)$ & $<0.001^{*}$ \\
\hline Glucose (mg/dl) & 325 & $105 \pm 53$ & $97(77-124)$ & $101 \pm 56$ & $112 \pm 49$ & 0.07 \\
\hline
\end{tabular}

$\mathrm{p}$ Values were calculated using Student $t$ test unless otherwise specified.

*Mann-Whitney U test.

$\mathrm{LDH}$, lactate dehydrogenase; WBC, white blood cell. 
Figure 1 Lymphocyte percentage in tuberculous pleurisy. (A) Distribution of cases by effusion lymphocyte percentage. (B) Generalised additive model analysis of relationship between lymphocyte percentage of pleural fluids and effect on probability of culture positivity of pleural fluids, which equals $\ln (\mathrm{p} / 1-\mathrm{p})$. Data are presented as fitted regression (line) and $95 \% \mathrm{Cl}$ (grey area).
A

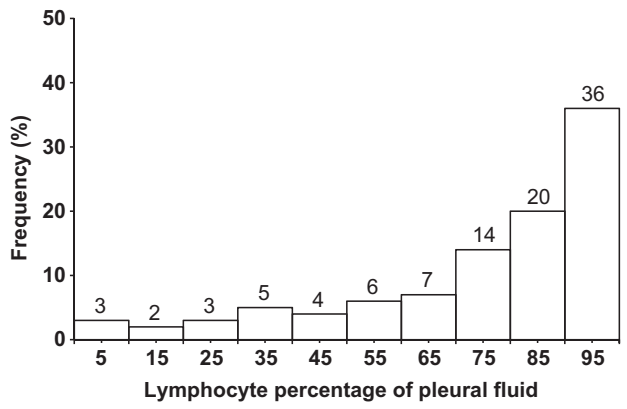

B

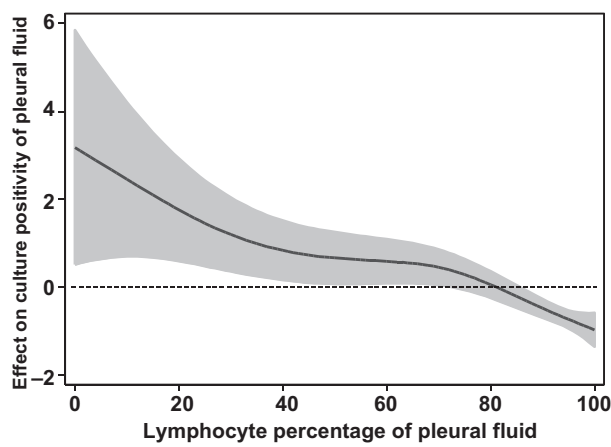

effusion culture using liquid culture media was $63 \%$ in this study cohort, which is much higher than the yield of less than $35 \%$ reported in most of the previous studies. ${ }^{523}$ The combination of the effusion and sputum cultures contributed to a good diagnostic yield of up to $79 \%$ compared with a yield of $74 \%$ in the histological examination of pleural biopsies. In addition, our study provides updated data about pleural fluid findings in tuberculous pleurisy (figure $1 \mathrm{~A}$ and table 2) and shows that the lymphocyte percentage of pleural fluid was negatively associated with the probability of a positive effusion culture (figure 1B). Furthermore, the differences between effusion culture-positive and culture-negative tuberculous pleurisy were highlighted in our study.

In this study, we cannot conclude that the combination of effusion and sputum cultures results in a higher yield than pleural biopsy because only 149 out of 382 patients received pleural biopsies, and the decision to perform a pleural biopsy may be a source of bias. Pleural biopsy is considered a reference procedure to diagnose tuberculous pleurisy due to a high yield and fast result. ${ }^{5}$ A prospective study showed a sensitivity of $66 \%$ and $100 \%$ for histology examination of closed needle and thoracoscopic pleural biopsy respectively. ${ }^{17}$ In 131 patients who received all examinations of pleural biopsy, effusion and sputum mycobacterial cultures in our study, pleural biopsy has the highest sensitivity (table 4). However, the patients receiving all

Table 3 Diagnostic yields of effusion culture, sputum culture and pleural biopsy for tuberculous pleurisy

\begin{tabular}{lr}
\hline Diagnostic procedures & n (\%) \\
\hline Positive effusion culture & $241 / 382(63)$ \\
Positive sputum culture & $124 / 241(51)$ \\
Negative sputum culture & $89 / 241(37)$ \\
Sputum culture not performed & $28 / 241(12)$ \\
Negative effusion culture & $141 / 385(37)$ \\
Positive sputum culture & $60 / 141(43)$ \\
Negative sputum culture & $64 / 141(45)$ \\
Sputum culture not performed & $17 / 141(12)$ \\
Positive sputum culture & $184 / 382(48)$ \\
Positive sputum culture/sputum culture performed & $184 / 337(55)$ \\
Positive AFB smear/positive sputum culture & $39 / 184(21)$ \\
Combining effusion and sputum cultures & $301 / 382(79)$ \\
Pleural biopsy performed & $149 / 382(39)$ \\
Observation of granuloma & $110 / 149(74)$ \\
Positive tissue culture/tissue culture performed & $41 / 104(39)$ \\
Either positive tissue culture or histology & $112 / 149(75)$ \\
\hline AFB, acid-fast bacillus.
\end{tabular}

three examinations may be a particular group in this retrospective study. The lymphocyte percentage of pleural fluid influenced the decision to perform a pleural biopsy and the positivity of effusion culture. Prospective studies are needed to clarify this issue. Furthermore, previous studies suggested that most patients with tuberculous pleurisy have more than $90 \%$ lymphocytes in their pleural fluid. ${ }^{7}{ }^{14}$ A highly cited study reported that only $6.7 \%$ of patients had fewer than $50 \%$ of lymphocytes in their pleural fluid. ${ }^{14}$ In contrast, the degree of lymphocyte predominance in our patients was lower than those of previous studies. This discrepancy may be partly related to the popular use of ultrasound for thoracentesis and early effusion studies. ${ }^{10}$

Tuberculous pleurisy may resolve spontaneously without treatment, but the patient frequently develops active $\mathrm{TB}$ at a later date. ${ }^{24} \mathrm{~A}$ pleural effusion that resolves with anti-TB therapy does not justify an accurate diagnosis of TB pleurisy. Thus the patients included in our study by the fourth criterion belong to a presumptive group of tuberculous pleurisy. However, removing these cases does not alter the main study findings but increases sensitivity of all diagnostic methods (online supplementary tables 1 and 2). In TB endemic areas, some patients with suspected tuberculous pleurisy prefer to receive a therapeutic trial with anti-TB therapy rather than undergo invasive pleural biopsy after malignancy and other common diseases have been excluded.

Mycobacterial culture of sputum has been suggested in the management of suspected tuberculous pleurisy, ${ }^{12}{ }^{25}$ though the

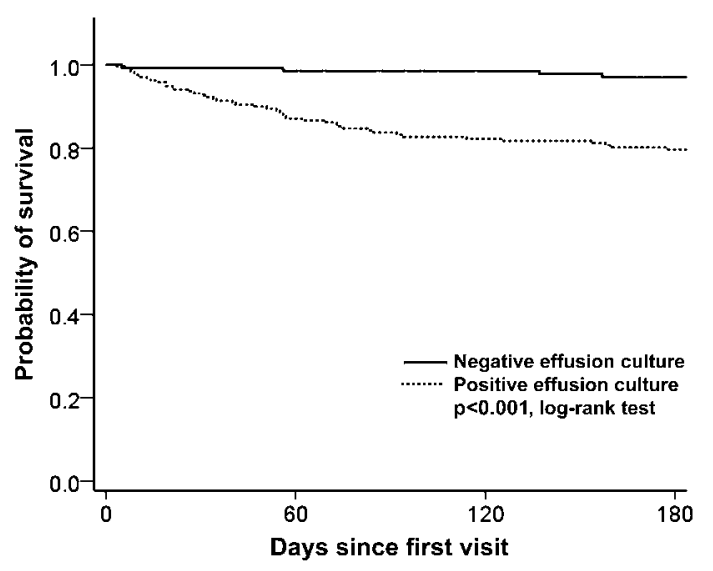

Figure 2 Kaplan-Meier estimates of survival for patients with positive and negative effusion cultures. 
Table 4 Comparison of diagnostic yields in 131 cases of tuberculous pleurisy having all data of pleural biopsy, effusion and sputum mycobacterial cultures

\begin{tabular}{ll}
\hline Diagnostic procedures & $\mathbf{n}(\%)$ \\
\hline Positive effusion culture & $70 / 131(53)$ \\
Positive sputum culture & $41 / 131(31)$ \\
Combining effusion and sputum cultures & $87 / 131(66)$ \\
Pleural biopsy revealing granuloma & $97 / 131(74)$ \\
\hline
\end{tabular}

sensitivity of sputum culture varied widely from $0 \%$ to $52 \%$ in previous studies. $^{12} 13$ Our results showed that $48 \%$ of patients had positive sputum cultures, which is comparable to previous studies with larger case numbers. ${ }^{8} 12$ Frequent lung parenchymal involvement in patients with tuberculous pleurisy shown in a CT study illustrated the rationale of using sputum cultures for the diagnosis of tuberculous pleurisy. ${ }^{26}$ Therefore, the combination of effusion and sputum cultures is a reasonable approach in the diagnosis of tuberculous pleurisy and may achieve a good diagnostic yield. Our study documented the high yield of this diagnostic approach. Another advantage of this approach over histological examination and other novel molecular tests is that it allows for susceptibility testing, which is important in areas with a high rate of drug resistance.

The diagnostic yield of the effusion cultures in our study was much higher than the reported values of $7-35 \%$ in most of the previous studies. 7121417 This discrepancy may be partly due to the difference in culture methods. Studies with low effusion culture yields mostly used solid culture media. However, liquid culture systems are known to have higher yields and faster results than those using solid media, especially for paucibacillary diseases, such as extrapulmonary $\mathrm{TB}^{27}{ }^{28}$ In addition, selection bias contributed to the varying yields of effusion culture in previous studies. Many prospective studies of tuberculous pleurisy excluded patients with multiple comorbidities and poor performance. However, patients who are immunocompromised are more likely to have a higher mycobacterial burden in the pleural cavity and, therefore, have a higher probability of a positive effusion culture. ${ }^{29}$ These exclusion criteria consequently decrease the yield of effusion culture. In addition, if neutrophilic pleural effusion is not considered indicative of tuberculous pleurisy and is excluded in a study, the sensitivity of the effusion culture also decreases. The negative association between lymphocyte percentage and effusion culture was well demonstrated in our study. Compared with previous studies, the selection bias was reduced in our study because of the comprehensive enrolment of all cases based on the backgrounds of a universal mycobacterial culture for all undiagnosed pleural effusions and a high index of suspicion due to endemic TB.

Our study also adds new insight to the pathogenesis of tuberculous pleurisy. The classical pathogenesis model, a rupture of subpleural caseous foci followed by a delayed hypersensitivity reaction to $M$ tuberculosis antigens, ${ }^{30-32}$ indicates two primary features of tuberculous pleurisy, including lymphocyte predominance and a low yield of effusion culture. Some investigators even suggested that tuberculous pleurisy was a disease caused by a hypersensitivity reaction rather than an infection of the pleura. ${ }^{14}{ }^{23}$ However, this hypothesis can be challenged if effusion lymphocytosis and a low yield of effusion culture are not universally present in every case of tuberculous pleurisy. Based on the findings of our study, we propose a new concept for tuberculous pleurisy. We hypothesise that tuberculous pleurisy is a continuous spectrum of disease (illustrated by online supplementary figure 1) rather than the two distinguishing clinical phenotypes of a delayed hypersensitivity reaction or true pleural infection. ${ }^{6} 33$ Tuberculous empyema represents an extreme condition, with a high percentage of neutrophils and a high mycobacterial load in pleural fluids. Previous studies have reported the presence of neutrophilic leukocytosis in tuberculous empyema and an increased neutrophil percentage in culturepositive tuberculous pleural effusions. ${ }^{34} 35$ Tuberculous parapneumonic effusion represents the common paucibacillary presentation of tuberculous pleurisy. The effusion lymphocyte percentage is negatively associated with the pleural mycobacterial burden and the likelihood of a positive effusion culture. It should be noted that this hypothesis is proposed solely based on the findings of our study and needs additional studies to test it.

The patients with positive effusion cultures were associated with a higher mortality rate in this study cohort (figure 2). However, a positive effusion culture was also associated with higher rates of cancer, renal failure, liver cirrhosis and a lower serum protein level. It is not clear whether the higher mortality rate in patients with positive effusion cultures was caused by a higher intrapleural mycobacterial burden or the poor health status of the host in this retrospective study. Further studies are needed to clarify the causality.

Our study has several limitations. This study was a retrospective analysis, and the effect of missing data on the results is unknown. In addition, this study was conducted in a TB endemic area and the results may not be generalisable to other areas.

In summary, our study yields several important findings that contribute to the current understanding of the diagnosis of tuberculous pleurisy. Using liquid culture media, the sensitivity of effusion culture was higher than has been previously reported. The combination of effusion and sputum cultures offers a good diagnostic yield and may be an alternative approach to diagnose tuberculous pleurisy in areas where pleural biopsy and other novel diagnostic tests are limited. Although further validation is needed, the concept of a continuous spectrum of tuberculous pleurisy offers a new and more practical consideration for this disease.

Acknowledgements The authors thank the TB case managers, Miss Y J Chien and S T Yang, for assistance with the study, and the members of the Taiwan AntiMycobacteria Investigation (TAMI) group for advice on interpretation of data.

Contributors SYR, YCC, JYW: study concept and design, analysis of data and drafting of manuscript. JWL: statistical analysis and interpretation of data. JYC, CTH, YWK: interpretation of data and review of manuscript. LNL, CJY: review of manuscript.

Funding The study was partly supported by National Taiwan University Hospital Yun-Lin Branch grant NTUHYL98.X007 and the Institute for Biotechnology and Medicine Industry of Taiwan.

\section{Competing interests None.}

Ethics approval The Research Ethics Committee of the National Taiwan University Hospital.

Provenance and peer review Not commissioned; externally peer reviewed.

Data sharing statement We would like to share the original data of our study. Dr J Y Wang is the corresponding author and has full access to all study data.

\section{REFERENCES}

1. Peto HM, Pratt RH, Harrington TA, et al. Epidemiology of extrapulmonary tuberculosis in the United States, 1993-2006. Clin Infect Dis 2009;49:1350-7.

2. Kruijshaar ME, Abubakar I. Increase in extrapulmonary tuberculosis in England and Wales 1999-2006. Thorax 2009:64:1090-5.

3. Liam CK, Lim KH, Wong CM. Causes of pleural exudates in a region with a high incidence of tuberculosis. Respirology 2000;5:33-8.

4. Valdes L, Alvarez D, Valle JM, et al. The etiology of pleural effusions in an area with high incidence of tuberculosis. Chest 1996;109:158-62. 
5. Light RW. Update on tuberculous pleural effusion. Respirology 2010:15:451-8.

6. Gopi A, Madhavan SM, Sharma SK, et al. Diagnosis and treatment of tuberculous pleural effusion in 2006. Chest 2007;131:880-9.

7. Berger HW, Mejia E. Tuberculous pleurisy. Chest 1973;63:88-92.

8. Seibert AF, Haynes J Jr, Middleton R, et al. Tuberculous pleural effusion. Twentyyear experience. Chest 1991;99:883-6.

9. Sibley JC. A study of 200 cases of tuberculous pleurisy with effusion. Am Rev Tuberc 1950;62:314-23.

10. Levine H, Szanto PB, Cugell DW. Tuberculous pleurisy. An acute illness. Arch Intern Med 1968; 122:329-32.

11. Rahman NM, Singanayagam A, Davies $\mathrm{HE}$, et al. Diagnostic accuracy, safety and utilisation of respiratory physician-delivered thoracic ultrasound. Thorax 2010;65:449-53.

12. Conde MB, Loivos AC, Rezende VM, et al. Yield of sputum induction in the diagnosis of pleural tuberculosis. Am J Respir Crit Care Med 2003;167:723-5.

13. Arrington CW, Hawkins $\mathrm{JA}$, Richert $\mathrm{JH}$, et al. Management of undiagnosed pleural effusions in positive tuberculin reactors. Am Rev Respir Dis 1966;93:587-93.

14. Valdes L, Alvarez D, San Jose E, et al. Tuberculous pleurisy: a study of 254 patients. Arch Intern Med 1998;158:2017-21.

15. Escudero Bueno C, Garcia Clemente M, Cuesta Castro B, et al. Cytologic and bacteriologic analysis of fluid and pleural biopsy specimens with Cope's needle. Study of 414 patients. Arch Intern Med 1990;150:1190-4.

16. Kumar S, Seshadri MS, Koshi G, et al. Diagnosing tuberculous pleural effusion: comparative sensitivity of mycobacterial culture and histopathology. Br Med J /Clin Res Ed) 1981;283:20.

17. Diacon AH, Van de Wal BW, Wyser C, et al. Diagnostic tools in tuberculous pleurisy: a direct comparative study. Eur Respir J 2003;22:589-91.

18. Somoskovi A, Magyar P. Comparison of the mycobacteria growth indicator tube with MB redox, Lowenstein-Jensen, and Middlebrook 7H11 media for recovery of mycobacteria in clinical specimens. J Clin Microbiol 1999;37:1366-9.

19. Cruciani M, Scarparo C, Malena M et al. Meta-analysis of BACTEC MGIT 960 and BACTEC 460 TB, with or without solid media, for detection of mycobacteria. J Clin Microbiol 2004;42:2321-5.

20. Taiwan Centers for Disease Control. Taiwan Tuberculosis Control Report 2009 Tainan City: Taiwan Centers for Disease Control, 2009.
21. Murray PR, Baron EJ. Manual of Clinical Microbiology. Washington, DC: ASM Press, 2003.

22. Nitta $\mathbf{H}$, Yamazaki S, Omori T, et al. An introduction to epidemiologic and statistical methods useful in environmental epidemiology. J Epidemiol 2010;20:177-84.

23. Ferrer J. Pleural tuberculosis. Eur Respir J 1997;10:942-7.

24. Roper WH, Waring JJ. Primary serofibrinous pleural effusion in military personnel. Am Rev Tuberc 1955;11:616-34.

25. Porcel JM. Tuberculous pleural effusion. Lung 2009;187:263-70.

26. Kim HJ, Lee HJ, Kwon SY, et al. The prevalence of pulmonary parenchymal tuberculosis in patients with tuberculous pleuritis. Chest 2006;129:1253-8.

27. Hillemann D, Richter E, Rusch-Gerdes S. Use of the BACTEC Mycobacteria Growth Indicator Tube 960 automated system for recovery of Mycobacteria from 9,558 extrapulmonary specimens, including urine samples. J Clin Microbiol 2006; 44:4014-17.

28. Lu D, Heeren B, Dunne WM. Comparison of the Automated Mycobacteria Growth Indicator Tube System (BACTEC 960/MGIT) with Lowenstein-Jensen medium for recovery of mycobacteria from clinical specimens. Am J Clin Pathol 2002; 118:542-5

29. Luzze H, Elliott AM, Joloba ML, et al. Evaluation of suspected tuberculous pleurisy: clinical and diagnostic findings in HIV-1-positive and HIV-negative adults in Uganda. Int J Tuberc Lung Dis 2001;5:746-53.

30. Stead WW, Eichenholz A, Stauss HK. Operative and pathologic findings in twentyfour patients with syndrome of idiopathic pleurisy with effusion, presumably tuberculous. Am Rev Tuberc 1955;71:473-502.

31. Allen JC, Apicella MA. Experimental pleural effusion as a manifestation of delayed hypersensitivity to tuberculin PPD. J Immunol 1968;101:481-7.

32. Leibowitz S, Kennedy L, Lessof MH. The tuberculin reaction in the pleural cavity and its suppression by antilymphocyte serum. Br J Exp Pathol 1973:54:152-62

33. Murray JF, Mason RJ. Murray and Nadel's Textbook of Respiratory Medicine. 5th edn. Philadelphia, PA: Saunders/Elsevier, 2010.

34. Bai KJ, Wu IH, Yu MC, et al. Tuberculous empyema. Respirology 1998;3:261-6.

35. Liu SF, Liu JW, Lin MC. Characteristics of patients suffering from tuberculous pleuritis with pleural effusion culture positive and negative for Mycobacterium tuberculosis, and risk factors for fatality. Int J Tuberc Lung Dis 2005; 9:111-15. 